\title{
Évolution de la digestibilité chez la vache Charolaise autour de la mise à l'herbe
}

\author{
J Agabriel, JM Giraud, M Petit, C Mauchamp \\ INRA Theix, station de recherches sur la lactation et l'élevage des ruminants, \\ 63122 Ceyrat, France
}

\begin{abstract}
Summary - Evolution of diet digestibility in Charolais cows changing from hay to grass diet. Six Charolais cows were individually fed hay $(1.5 \mathrm{~kg}$ dry matter $/ 100 \mathrm{~kg}$ live weight) for 14 days, then cut highland grass ad libitum for another 18 days. Organic matter digestibility (OMD) of the diet was measured each day. OMD of the hay diet was $55.3 \%$. Grass digestibility $(74.0 \%)$ was obtained within 4 days. Large variations in OMD were observed between animals during the 4 days.
\end{abstract}

Le changement brusque, à la fois de milieu et de régime almentaire correspondant à la mise à l'herbe, influe fortement sur les performances des animaux et notamment sur la reprise de la masse corporelle des vaches allaitantes. La transition foin-herbe nécessite d'être détaillée sous l'aspect nutritionnel, en particulier la vitesse de la mise en place de la digestibilité.

Matériel et méthodes - Six vaches charolaises au $3^{\circ}$ mois de lactation pesant en moyenne $617 \mathrm{~kg}$, sont placées en stalles à digestibilité le 15 mai. Elles reçoivent d'abord pendant $14 \mathrm{j}$ du foin (50\% fléole et $50 \%$ dactyle) distribué à raison de $1,5 \mathrm{~kg}$ de matière sèche (MS) par $100 \mathrm{~kg}$ de poids, $1 \mathrm{~kg}$ de tourteau de soja et $0,5 \mathrm{~kg}$ de maîs grain. À partir du 30 mai (jour J1), pour simuler la mise à l'herbe, elles ne reçoivent plus que de l'herbe coupée distribuée à volonté 5 fois dans la journée. Cette herbe provient d'une prairie permanente de montagne (1 $100 \mathrm{~m})$ à dominance de fléole exploitée du stade pâturage au stade épiaison. La mesure de la digestibilité sur les vaches continue jusqu'au 17 juin. Parallèlement, depuis le 25 mai, la digestibilité de cette herbe est aussi mesurée sur un lot de 6 moutons castrés, maintenus en cage et alimentés à volonté.

Résultats et discussion - En 3 jours, les quantités de MS d'herbe ingérée (QI) par les vaches atteignent leur maximum, passant de $11,8 \pm 0,3$ et 10,2 $\pm 0,8 \mathrm{~kg}$ MS les 2 premiers j (J1 et J2), à $12,2 \pm 0,5$ le jour J3 puis à 16,2 \pm $0,1 \mathrm{~kg}$ MS le jour J4. Les 6 vaches atteignent le même jour ce maximum, puis les Ql se stabilisent autour de la moyenne (15,8 kg MS) les 2 semaines suivantes.

À l'inverse, les quantités de fèces excrétées ne varient pratiquement pas entre le régime foin (moyenne 5,2 \pm $0,3 \mathrm{~kg} \mathrm{MS}$ ) et le régime herbe (5,0 \pm $0,3)$ : elles n'augmentent légèrement qu'en $\mathrm{J} 2$, J3 et $\mathrm{J} 4(5,5 \mathrm{~kg} \mathrm{MS})$. Mais leurs teneurs en MS diminuent forte- 
ment passant de $11,7 \% \pm 0,7$ (J1) à $6,1 \% \pm 0,4$ au $8^{e}$ jour, et ne se stabilisent qu'en $2^{\ominus}$ semaine : $8,0 \% \pm 0,4$ en moyenne.

La digestibilité apparente de la matière organique (DMO) (fig 1) a peu varié autour de la moyenne sur le régime foin $(55,3 \%)$. Si l'on rapporte les fèces à la MS ingérée la veille, elle passe ensuite par un minimum en $\mathrm{J} 2$ puis s'accroît brusquement de 17 points en 3 j pour atteindre $74,0 \%$ en J5. Elle s'approche alors fortement de la DMO mesurée parallèlement sur les moutons : $76,4 \%$.

Les réactions individuelles après le début de la distribution d'herbe ne sont pas homogènes: en $\mathrm{J} 1$, le coefficient de variation (CV) de la DMO est limité $(C V=4,7 \%)$ et comparable à celui des

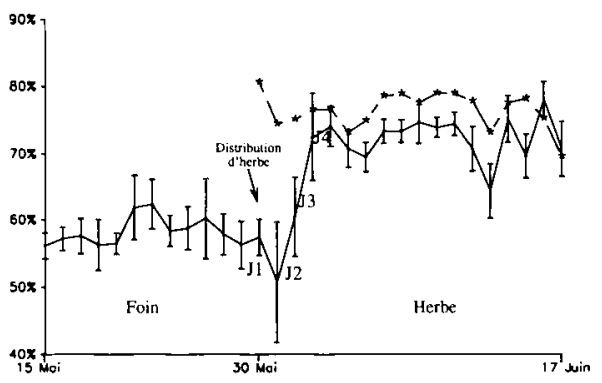

Fig 1. Évolution et variabilité de la digestibilité de la matière organique d'un régime "foin "à un régime " herbe". Vaches : moutons : *-*. jours précédents sur le régime foin ( $\mathrm{CV}$ $=5,7 \%$ en moyenne); en $\mathrm{J} 2$ il passe par un maximum à $17 \%$, et c'est en $\mathrm{J} 5$ qu'il revient à un niveau comparable au niveau initial ( $C V=4,0 \%$ ).

On peut donc estimer que la durée totale de la période de transition digestive entre les 2 régimes est de $4 \mathrm{j}$ environ pour toutes les vaches.

En effet, le temps de séjour moyen du foin dans le tractus digestif est long ( $75 \mathrm{~h}$ : Bines et al, 1969, $55 \mathrm{~h}$ : Poncet et al, 1987), et l'excrétion dans les fèces de son indigestible se prolonge donc sur le régime "herbe», ce qui amène à sous-estimer la digestibilité de cette dernière.

En conclusion, dans de bonnes conditions, le passage d'un régime constitué de foin à un régime herbe ne pose pas de problèmes particuliers aux vaches, qui s'adaptent en moins de $5 \mathrm{j}$. Ceci explique la rapidité de la reprise de poids ou de l'augmentation de production laitière dès la mise à l'herbe (Coulon et al, 1988). Toutefois la variabilité individuelle est élevée.

Bines JA, Suzuki S, Balch CC (1969) $\mathrm{Br} J$ Nutr 23, 695-704

Coulon JB, D'hour P, Petit M (1988) Livest Prod Sci 20, 119-134

Poncet C, Gomez L, Michalet-Doreau B, Geay Y (1987) Reprod Nutr Dev 27 (1B), 219-220 